\title{
Anti-quorum sensing activity of quercetin in combination with imipenem and ciprofloxacin against Pseudomonas aeruginosa PA01
}

\section{Tabasom Taghavi}

Zanjan University of Medical Sciences

Habib Zeighami

Zanjan University of Medical Sciences

\section{Azam Heidari}

Zanjan University of Medical Sciences

\section{Mina Gholami}

Zanjan University of Medical Sciences

\section{Farzaneh Rafiee}

Zanjan University of Medical Sciences

\section{Fakhri Haghi ( $\nabla$ haghi@zums.ac.ir)}

Zanajn University of Medical Sciences

\section{Research}

Keywords: Ciprofloxacin, Imipenem, Pseudomonas aeruginosa, Quercetin, Quorum sensing

Posted Date: April 20th, 2021

DOI: https://doi.org/10.21203/rs.3.rs-435536/v1

License: (1) This work is licensed under a Creative Commons Attribution 4.0 International License. Read Full License 


\section{Abstract \\ Background}

The inhibition of quorum sensing (QS) is considered as a novel strategy for development of antipathogenic agents and management of nosocomial infections caused by Pseudomonas aeruginosa. The present study aimed to investigate the anti-quorum sensing activity of sub-MIC concentrations of quercetin (Q), imipenem (IPM) and ciprofloxacin (CIP) alone and in combination against $P$. aeruginosa.

\section{Methods}

We determined the MIC and synergistic activity of Q, IPM and CIP against $P$. aeruginosa PAO1. The effect of sub-MIC (1/4 and 1/16 MIC) concentrations of Q, IPM and CIP alone and in combination on QS related virulence factors and expression of las/ and las $R$ genes was also determined.

\section{Results}

The addition of Q decreased the MIC of IPM and CIP and there was additive effect from these combinations. Quercetin did not show significant effects on cell growth at concentrations of $1 / 4$ and 1/16 MIC. However, sub-MIC concentrations (1/4 and 1/16 MIC) of Q in combination with IPM or CIP showed the significant reduction in QS dependent phenotypes $(P<0.001)$. The combination of Q with IPM or CIP at the concentrations of 1/4 MIC showed the greatest reduction in the expression levels of las/ compared with untreated control $(P<0.001)$. The docking results suggested that quercetin has potential to inhibit the LasR protein.

\section{Conclusion}

Considering the beneficial biological effects of quercetin and its inhibitory effects on QS related virulence traits, the combination of this natural compound with imipenem or ciprofloxacin may be used as an effective approach for managing of multidrug resistant infections of $P$. aeruginosa.

\section{Background}

Pseudomonas aeruginosa is a leading cause of nosocomial infections with high mortality rate due to the limited therapeutic options [1]. Resistance to different antimicrobial agents and survival ability in various hospital environments have made it as a major cause of nosocomial infections worldwide [2]. The multidrug resistant (MDR) P. aeruginosa has become a serious problem in healthcare settings worldwide especially in Spain, Italy, Brazil, South Korea and USA [1]. Biofilm formation on biotic and abiotic surfaces is another important factor contributing to chronic and persistent $P$. aeruginosa infections [3]. Conventional antimicrobials are usually inefficient in the treatment of biofilm-associated infections [3]. 
Heterogeneous populations of biofilms communicate by chemical molecules and cell to cell signaling play an important role in control of virulence factors and antibiotic resistance [4]. Therefore, the development of new therapeutic approaches is necessary to prevent infections caused by antibiotic resistant strains [5]. The inhibition of quorum sensing (QS) is considered as a novel strategy for development of antipathogenic agents since it is involved in several processes including virulence, motility, sporulation, biofilm formation, genetic competency, antibiotic and proteolytic enzyme production [5-7]. QS in P. aeruginosa consists of four systems including Lasl/LasR, RhII/RhIR, PQS/MvfR and IQS mediated through small diffusible signal molecules of the $\mathrm{N}$-acyl homoserine lactones (AHL) family called autoinducers (Als) [6]. These Als are involved in exoenzymes production, bacterial adhesion, biofilm formation and regulation of several virulence factors $[7,8]$. The Lasl/R system regulates the exotoxin A, LasA protease, LasB elastase and Apr alkaline protease production through 3-OH-C12homoserine lactone. While Rhll/R system regulates the production of rhamnolipids, pyocyanin, hydrogen cyanide, and cytotoxic lectins through C4-homoserine lactone [9]. Therefore, quorum sensing inhibitors (QSIs) can be used to attenuate the virulence and pathogenesis and may have a role in control and treatment of acute and persistent infections $[3,10]$. The QSIs can be inhibiting the signal generator, degrade the signal molecule or blockage the signal receptor [3]. Various natural and chemically synthesized quorum sensing inhibitors have been reported [6, 7]. However, the limitation of the use of these compounds in the food industry and mammalian cells has led to the search of novel QSIs [6]. Quercetin is a typical flavonoid widely distributed in nature. It is found commonly in many fruits, vegetables, leaves and grains [6]. Several investigations have reported the anti-microbial, anti-quorum sensing, anti-oxidant, anti-carcinogenic, anti-inflammatory, anti-apoptotic and anti-histamine effects of quercetin $[4,6,11,12]$. One strategy that can be useful in the treatment of MDR $P$. aeruginosa infections is the synergy using combinations of natural compounds with antibiotics leading to enhancement of antibacterial activity of antibiotics. Previous investigations have been carried out on the biological activities of quercetin but the combination effects of this natural compound with ciprofloxacin or imipenem on $P$. aeruginosa QS have not been demonstrated $[4,6]$. So, the present study aimed to investigate the anti-quorum sensing activity of sub-MIC concentrations of quercetin, imipenem and ciprofloxacin alone and in combination against $P$. aeruginosa. To understand the mechanism of QSI activity, the molecular docking was carried out to show the possible interactions of quercetin with the LasR receptor protein.

\section{Methods}

\section{Bacterial strains, growth media and conditions}

Wild type $P$. aeruginosa PA01 was used for the assay of QSI activity of quercetin, imipenem and ciprofloxacin. Chromobacterium violaceum CV026 was used as a reporter strain. The cultures were grown in Luria-Bertani (LB) broth/agar (Merck, Darmstadt, Germany) aerobically at $37^{\circ} \mathrm{C}$ or $30^{\circ} \mathrm{C}$ for $16-18 \mathrm{~h}$. Strains were preserved at $-80^{\circ} \mathrm{C}$ in Tryptic Soy Broth (TSB, Merck, Darmstadt, Germany) containing $10 \%$ $(\mathrm{v} / \mathrm{v})$ glycerol. 


\section{Materials}

Quercetin, imipenem and ciprofloxacin were purchased from Sigma-Aldrich (Buchs, Switzerland) and stock solutions prepared according to the Clinical and Laboratory Standards Institute (CLSI) criteria [13]. Fresh stock solution of quercetin was prepared in dimethyl sulfoxide (DMSO, 99.9\%; Sigma-Aldrich; Buchs, Switzerland) at the concentration of $10 \mathrm{mg} / \mathrm{ml}$. The final concentration of imipenem and ciprofloxacin stock solutions was $1 \mathrm{mg} / \mathrm{ml}$. N-Acyl-homoserine lactone (C6-HSL) (Sigma-Aldrich; Buchs, Switzerland) was used at $20 \mu \mathrm{M}$ in C. violaceum CV026 biosensor bioassay.

\section{Determination of minimum inhibitory concentration}

The broth microdilution method was used to determine the minimum inhibitory concentration (MIC) of imipenem, ciprofloxacin and quercetin [13]. Twofold serial dilutions of imipenem, ciprofloxacin $(256,128$, $64,32,16,8,4,2,1,0.5,0.25,0.125,0.06,0.03 \mu \mathrm{g} / \mathrm{ml})$, and quercetin $(500,250,125,62.5,31.2,15.6,7.8$, $3.9,1.9,0.9 \mu \mathrm{g} / \mathrm{ml}$ ) were prepared and then inoculated with $\mathrm{PAO}_{1}$ overnight culture containing $5 \times 10^{6}$ $\mathrm{CFU} / \mathrm{ml}$ and incubated at $37^{\circ} \mathrm{C}$ for $24 \mathrm{~h}$. Minimum inhibitory concentration was calculated as the lowest concentration that inhibited visible bacterial growth.

Checkerboard titration method was used to assessed the synergistic activity of quercetin with imipenem or ciprofloxacin as previously described [14]. The fractional inhibitory concentration (FIC) index was determined as follows: FIC index = FIC A + FIC B.

$\mathrm{FIC}$ of quercetin $(\mathrm{FIC} \mathrm{A})=$ concentration of quercetin in combination/MIC of quercetin alone;

FIC of antibiotics (FIC B) $=$ MIC of antibiotics (imipenem/ciprofloxacin) in combination/MIC of antibiotics alone.

The obtained FIC index was interpreted as follows: $<0.5$, synergy; 0.5 to 0.75 , partial synergy; 0.76 to 1.0 , additive effect; $>1.0$ to 4.0 , indifference; and $>4.0$, antagonism [6].

\section{Cell growth analysis}

The growth of treated and untreated PA01 was measured using the broth microdilution method [13]. Luria-Bertani broth containing different concentrations of quercetin, imipenem and ciprofloxacin alone and in combination $(0.015 \times$ MIC to $4 \times$ MIC) was inoculated with overnight culture of PAO1 and incubated at $37^{\circ} \mathrm{C}$ for $16 \mathrm{~h}$. Optical density of treated and untreated cultures was measured and the percentage of cell growth calculated as follow: $\mathrm{OD}_{630}$ (treated $\left.\mathrm{PAO}_{1}\right) / \mathrm{OD}_{630}$ (untreated $\left.\mathrm{PAO}_{1}\right) \times 100$ [15].

\section{Biosensor bioassay}

To demonstrate the anti-QS activity of quercetin, imipenem and ciprofloxacin alone and in combination, the well diffusion was used by the reporter strain $C$. violaceum CV026 [15]. Fifty microliters of compounds at the concentrations of 1/4 and 1/16 MIC were loaded onto wells on the surface of CV026 
inoculated LB agar plates supplemented with $20 \mu \mathrm{M}$ of C6-HSL and incubated at $30^{\circ} \mathrm{C}$ for $24 \mathrm{~h}$. The presence of a pigmentless zone of viable cells around the well was considered as QS inhibition.

\section{Effects on Biofilm formation}

The effect of quercetin, imipenem and ciprofloxacin alone and in combination on biofilm formation of PAO1 was assessed using microtiter plate [16]. Luria-Bertani broth with and without sub-MIC concentrations (1/4 and 1/16 MIC) of compounds were inoculated with overnight cultures of $\mathrm{PAO}_{1}$ and incubated at $37^{\circ} \mathrm{C}$. After $24 \mathrm{~h}$ of incubation, biofilms were washed three times with sterile PBS, fixed with $150 \mu$ l of $99 \%(\mathrm{v} / \mathrm{v})$ methanol and stained with $0.2 \%(\mathrm{w} / \mathrm{v})$ crystal violet (HiMedia, India). Finally, 33\% (v/v) glacial acetic acid was used to solubilize crystal violet. The absorbance of treated and untreated samples was measured at $\mathrm{OD}_{590} \mathrm{~nm}$. The percentage of biofilm inhibition was calculated as follow: $\left(1-\mathrm{OD}_{590}\right.$ of treated $\mathrm{PAO}_{1} / \mathrm{OD}_{590}$ of untreated $\left.\mathrm{PAO}_{1}\right) \times 100$. Reported values are the mean of three measurements.

\section{Motility assays}

Swarming and twitching motilities were assayed on agar plates with or without a sub-MIC concentration of quercetin, imipenem and ciprofloxacin alone and in combination as described previously [17].

\section{Effects on alginate production}

The effect of quercetin, imipenem and ciprofloxacin alone and in combination on the alginate production of PAO1 was assayed according to previous studies $(15,18)$. Briefly, $5 \mathrm{ml}$ of LB broth with or without subMIC concentrations (1/4 and 1/16 MIC) of compounds were inoculated with $500 \mu$ of PAO1 suspension $\left(1 \times 10^{8} \mathrm{CFU} / \mathrm{ml}\right)$ and incubated at $37^{\circ} \mathrm{C}$. After $24 \mathrm{~h}$ of incubation, $1 \mathrm{ml}$ of culture was centrifuged at 12000 $\mathrm{rpm}$ for $30 \mathrm{~min}$ and the supernatant maintained at $80^{\circ} \mathrm{C}$ for $30 \mathrm{~min}$. The centrifuged supernatant was precipitated with ice-chilled ethanol $99 \%(\mathrm{v} / \mathrm{v})$ at $4{ }^{\circ} \mathrm{C}$ for $2 \mathrm{~h}$ and mixed with $1 \mathrm{ml}$ of sterile saline $(0.9 \%)$. Then, one $\mathrm{ml}$ of borate sulfuric acid reagent $\left(100 \mathrm{mM} \mathrm{H}_{3} \mathrm{BO}_{3}\right.$ in concentrated $\left.\mathrm{H}_{2} \mathrm{SO}_{4}\right)$ and $34 \mu \mathrm{l}$ of carbazole reagent $(0.1 \%$ in ethanol) were added to $118 \mu$ l of sample on ice. The mixture was heated for $30 \mathrm{~min}$ at $55^{\circ} \mathrm{C}$ and the absorbance at $\mathrm{OD}_{530} \mathrm{~nm}$ was measured.

\section{Effects on pyocyanin production and hydrogen peroxide susceptibility}

The effect of quercetin, imipenem and ciprofloxacin alone and in combination on pyocyanin production and hydrogen peroxide $\left(\mathrm{H}_{2} \mathrm{O}_{2}\right)$ susceptibility of PAO1 was assessed according to previous studies $[15,18$, 19].

\section{RNA extraction and cDNA synthesis}

Total RNA of cultivated $\mathrm{PAO}_{1}$ with and without sub-MIC concentrations (1/4 and 1/16 MIC) of quercetin, imipenem and ciprofloxacin alone and in combination was extracted using EZ-10 Spin Column Total RNA Miniprep Super Kit (Bio Basic, Canada) with on-column DNasel digestion (Bio Basic, Canada) according to the kit handbook. 
The purity and concentration of RNA samples were determined by a NanoDrop Spectrophotometer (ND1000, Nano-Drop Technologies, Wilmington, DE). Complementary DNA (cDNA) was then synthesized using PrimeScript ${ }^{\mathrm{TM}}$ RT reagent Kit (Takara, Japan). Reverse transcription was performed in a reaction mixture with total volume of $10 \mu \mathrm{l}$ containing $2 \mu \mathrm{l}$ of $5 \mathrm{X}$ PrimeScript Buffer, $0.5 \mu \mathrm{l}$ of PrimeScript RT Enzyme Mix I, $0.5 \mu$ l of Random 6 mers $(100 \mu \mathrm{M})$, 500ng of RNA and RNase free water to complete the volume. The reactions were incubated at $37^{\circ} \mathrm{C}$ for $30 \mathrm{~min}, 85^{\circ} \mathrm{C}$ for $5 \mathrm{sec}$ and $4^{\circ} \mathrm{C}$ for $10 \mathrm{~min}$.

\section{Quantitative Real Time PCR}

The effect of sub-MIC concentrations (1/4 and 1/16 MIC) of quercetin, imipenem and ciprofloxacin alone and in combination expression of quorum sensing genes las/ and las $R$ was evaluated as described previously $[16,17]$. Real time PCR was performed in a reaction mixture with total volume of $20 \mu \mathrm{l}$ containing $10 \mu$ l of TB Green Premix Ex Taq (Takara, Japan), $0.4 \mu$ l of each primer $(10 \mu \mathrm{M}), 0.4 \mu$ l of ROX Reference Dye (50X), $1 \mu \mathrm{l}$ of cDNA (100ng) and $7.8 \mu$ l sterile purified water to complete the volume. Assays were performed in triplicate with an Applied Biosystems StepOnePlus ${ }^{\text {TM }}$ Real-Time PCR System. The expression of the target genes was normalized to the expression of reference gene oprL (encoding the outer membrane protein). Melting curve analysis demonstrated that the accumulation of TB Green-bound DNA was target gene specific. The no template control (NTC) and no reverse transcriptase control (no-RT) were included in all experiments. The expression of cultures grown in the presence of sub-MIC concentrations of compounds were compared with untreated cultures and the data were analysed using the $2^{-\Delta \Delta C t}$ method [20].

\section{Molecular Docking}

In order to study of possible interactions of quercetin with LasR, high resolution X-ray crystallized structure of $P$. aeruginosa LasR protein (PDB ID: 2UV0) was obtained from the RCSB Protein Data Bank and molecular docking analysis was performed using Auto Dock Tools (ADT, version 4.2.6) [21]. The 3D crystal structure of protein was prepared for docking by removing the co-crystallized ligand and waters from PDB file of $2 \mathrm{UV} 0$. Also, the ligand was prepared by energy minimization using the semi-emperical method of AM1 and molecular mechanics force field of MM + with Hyperchem Professional 8.0 program. Gasteiger method was used for partial charges calculation and the grid box was settled to include all amino acid residues in the active site. After completion of docking, the best generated conformer of quercetin was selected based on docking score for further analysis. The graphical representations were shown using UCSF Chimera 1.10.2 program. The accuracy of docking method for the LasR receptor system was calculated through root mean square distance (RMSD) value between the co-crystallized inhibitor (3-oxo- $\mathrm{C}_{12}-\mathrm{HSL}$ ) conformation and the best docked pose of native ligand.

\section{Statistical analysis}

The data were analyzed with SPSS version 17.0 software (SPSS, Inc., Chicago, IL) and expressed as means and standard deviations of $\Delta \mathrm{C}_{\mathrm{t}}$ values. The One Way ANOVA (Tukey) test was used to determine the statistical significance of the data. A $P$ value of $<0.05$ was considered significant. 


\section{Results}

\section{Determination of minimum inhibitory concentration}

The MIC of quercetin, imipenem and ciprofloxacin against PAO1 was found to be $62.5,1$ and 0.125 $\mu \mathrm{g} / \mathrm{ml}$, respectively. Therefore, further experiments were carried out at sub-MIC concentrations $(1 / 4$ and $1 / 16 \mathrm{MIC})$.

The synergistic effect of quercetin with imipenem or ciprofloxacin was determined using checkerboard titration method. The addition of quercetin decreased the MIC of imipenem and ciprofloxacin by 3 fold. The FIC index of each combination is shown in Table 1. It reflects that FIC index was 0.87 and 0.86 when quercetin was combined with imipenem or ciprofloxacin, respectively. There was additive effect from quercetin and ciprofloxacin or imipenem combinations.

Table 1

MIC determination and effect of quercetin in combination with imipenem and ciprofloxacin.

\begin{tabular}{|lllll|}
\hline Treatment groups & Imipenem & FIC index & Ciprofloxacin & FIC index \\
\hline Control $(\mu \mathrm{g} / \mathrm{ml})$ & 1 & & 0.125 & \\
\hline Quercetin added $(\mu \mathrm{g} / \mathrm{ml})$ & 0.32 & 0.87 & 0.03 & 0.86 \\
\hline
\end{tabular}

\section{Cell growth analysis}

The effect of concentrations of $0.015 \times$ MIC to $4 \times$ MIC of quercetin, imipenem and ciprofloxacin alone and in combination on cell growth of PAO1 is shown in Fig. 1. The concentration of $1 \times$ MIC of quercetin decreased the cell growth to $18.1 \%$ in comparison with untreated PA01. However, the combination of quercetin with imipenem or ciprofloxacin at concentrations of $1 \times$ MIC significantly decreased the cell growth to $8.3 \%$ and $5.05 \%$, respectively $(P<0.001)$. The combination of quercetin with imipenem or ciprofloxacin at the concentrations of $1 / 2$ and $1 / 4$ MIC inhibited the cell growth compared with untreated control (ranging from 12.2-69.7\%). The cell growth was not significantly reduced at the concentrations of 1/16 MIC of these compounds (Fig. 1).

\section{Biosensor bioassay}

Quercetin, imipenem and ciprofloxacin showed anti-quorum sensing activity in $C$. violaceum CV026 biosensor bioassay. The concentrations of $1 / 4$ and 1/16 MIC of quercetin showed the pigmentless zones of 15 and $9 \mathrm{~mm}$, respectively, indicating the violacein inhibition around the wells. The combination of quercetin with imipenem at the concentrations of 1/4 and 1/16 MIC showed stronger anti-quorum sensing activity with pigmentless zones of 24 and $17 \mathrm{~mm}$, respectively. Furthermore, pigmentless zones of 21 and $16 \mathrm{~mm}$ were observed at the concentrations of 1/4 and 1/16 MIC of quercetin in combination with ciprofloxacin.

\section{Effects on Biofilm formation}


The biofilm formation was inhibited in the presence of quercetin, imipenem and ciprofloxacin alone and in combination between $15-84 \%$ (Fig. 2). The inhibitory effect was concentration dependent. The concentration of $1 / 4 \mathrm{MIC}$ of quercetin inhibited the biofilm formation by $70 \%$ in comparison with untreated PAO1 $(P<0.05)$. The combination of quercetin with imipenem or ciprofloxacin at the concentrations of $1 / 4 \mathrm{MIC}$ showed the greatest inhibitory effect on biofilm formation with $84 \%$ reduction $(P<0.001)$.

\section{Effects on motility}

The effect of quercetin, imipenem and ciprofloxacin alone and in combination on motility of PAO1 is shown in Fig. 3. There was a 50\% decrease in twitching and $33.3 \%$ decrease in swarming motility in the presence of quercetin at the concentration of 1/4 MIC. However, there was no significant effect on swarming motility at $1 / 16 \mathrm{MIC}$ of quercetin. The combination of quercetin with imipenem or ciprofloxacin at the concentrations of $1 / 4 \mathrm{MIC}$ significantly decreased twitching and swarming motilities (ranged from $45.4-55 \%)(P<0.05)$. The combination of quercetin with imipenem or ciprofloxacin at the concentration of $1 / 4$ MIC was more effective than these compounds alone.

\section{Effects on alginate production}

The concentrations of $1 / 4$ and $1 / 16$ MIC of quercetin, imipenem and ciprofloxacin alone and in combination exhibited $15.8-78.9 \%$ reduction in alginate production compared with untreated PAO1 (Fig. 4). The combination of quercetin with ciprofloxacin exhibited $78.9 \%$ decrease in alginate production at the concentration of $1 / 4 \mathrm{MIC}(P<0.001)$. Similar effect was observed for quercetin and imipenem combination with $73.7 \%$ reduction in alginate production in comparison with untreated control $(P<$ 0.001).

\section{Effects on pyocyanin production}

The effect of quercetin, imipenem and ciprofloxacin alone and in combination on pyocyanin production is shown in Fig. 5. There was $26.8-74.6 \%$ decrease in pyocyanin production in the presence of quercetin, imipenem and ciprofloxacin alone and in combination. The concentration of $1 / 4$ MIC of quercetin decreased the pyocyanin production by $47.2 \%$ in comparison with untreated PA01. Furthermore, the combination of quercetin with imipenem or ciprofloxacin at the concentrations of $1 / 4$ MIC significantly inhibited the pyocyanin production compared with untreated PAO1 (74.6\% and $60.9 \%$, respectively) $(P<$ 0.001).

\section{Effects on hydrogen peroxide susceptibility}

The concentrations of $1 / 4 \mathrm{MIC}$ of quercetin, imipenem and ciprofloxacin significantly increased the sensivity of PAO1 to $\mathrm{H}_{2} \mathrm{O}_{2}$ (ranged from 1.8 to 2 fold) $(P<0.05)$. The combination of quercetin with imipenem or ciprofloxacin at the concentrations of $1 / 4 \mathrm{MIC}$ showed the greatest sensivity to $\mathrm{H}_{2} \mathrm{O}_{2}$ by 2.4 and 2.5 fold, respectively $(P<0.05)$ (Fig. 6).

\section{Expression of quorum sensing regulated genes}


Relative expression of quorum sensing regulated genes las/ and las $R$ was measured from calculated Ct values and standard curves. They showed the same melting profiles with no primer dimer formation. The standard curve of reference gene oprL and target genes las/ and lasR showed R2 values $0.99-0.97$. Relative expression levels of treated cultures were compared with untreated cultures and the data were analyzed using the $2^{-\Delta \Delta C t}$ method. Changes in expression level were shown in Fig. 7. Quercetin, imipenem and ciprofloxacin alone and in combination significantly repressed the expression of las/and las $R$ between $65-95 \%$ relative to untreated PAO1 $(P<0.001)$. The combination of quercetin with imipenem or ciprofloxacin at the concentrations of 1/4 MIC showed the greatest reduction in the expression levels of lasl, $95 \%$ and $92 \%$ respectively, compared with untreated control $(P<0.001)$.

\section{Molecular docking}

In order to study the interactions of quercetin with LasR, it was necessary to identify the binding site of this compound in the QS regulatory protein. As shown in Fig. 8, quercetin made hydrogen bonding interactions with Arg 61, Leu 110, Trp 60, and Tyr 93 with binding energy of $-8.68 \mathrm{kcal} / \mathrm{mol}$ in the active site of LasR. Furthermore, the overlay of the native ligand (3-oxo- $\left.\mathrm{C}_{12}-\mathrm{HSL}\right)$ orientation with quercetin in the active site of LasR was illustrated in Fig. 9. The RMSD value of the docked conformer relative to its crystal orientation was found $0.84 \AA$ that proposing good docking reliability [22].

\section{Discussion}

Quorum sensing inhibition is considered as a novel strategy for development of antipathogenic agents and control of microbial infections [5-7]. The effect of sub-MIC concentrations of various natural and chemically synthesized compounds has been investigated on the expression of quorum sensing regulated genes $[16,17]$, virulence traits $[7,15]$ and biofilm formation $[19,23]$ in $P$. aeruginosa. We investigated anti-quorum sensing activity of sub-MIC concentrations of quercetin, imipenem and ciprofloxacin alone and in combination against $P$. aeruginosa. According to our results, the addition of quercetin decreased the MIC of imipenem and ciprofloxacin and there was additive effect from these combinations. In our previous study, sub-MIC concentrations of curcumin in combination with ciprofloxacin also showed an additive effect [16]. The combination of quercetin with imipenem or ciprofloxacin significantly decreased the cell growth of PAO1 at concentrations of $1 \times$ MIC. However, the cell growth was not significantly reduced at concentrations of $1 / 4$ and 1/16 MIC of these combinations, proving that their anti-quorum sensing effects were achieved through inhibition of QS not by killing of cells. Similar results were reported by El-Mowafy et al [7] which showed that sub-MIC concentrations of aspirin significantly decreased the quorum sensing signals of $P$. aeruginosa without reduction in cell growth [7]. In the lack of significant effects of quercetin on the cell growth at concentrations of $1 / 4$ and $1 / 16$ MIC, it has been the capacity to inhibit quorum sensing system in C. violaceum CV026 biosensor bioassay. Quercetin exhibited a concentration-dependent reduction in violacein production, indicated by the zone of pigmentless around the well. However, the combination of quercetin with imipenem at concentrations of 1/4 MIC showed stronger anti-quorum sensing activity in CV026. Similarly, subinhibitory concentrations of natural plant extracts such as curcumin, cinnamaldehyde and its 
derivatives, iberin, furanone and its derivatives, ajoene, catachin and some antibiotics as azithromycin, ceftazidime and ciprofloxacin significantly inhibit QS signaling $[3,7,16,17,24]$. In study conducted by Gopu et al [6] quorum quenching potential of quercetin was reported against $C$. violaceum CV026. According to their results, quercetin showed the significant reduction in quorum sensing dependent phenotypes like violacein production and biofilm formation at the concentration of $80 \mu \mathrm{g} / \mathrm{ml}$ [6]. However, we showed the violacein inhibition in $C$. violaceum CV026 by the lower concentrations of quercetin (15.6 and $3.9 \mu \mathrm{g} / \mathrm{ml}$ ).

Biofilm formation is an important factor contributing to pathogenicity, antibacterial resistance and development of chronic and persistent $P$. aeruginosa infections $[3,7]$. Since quorum sensing is one of the crucial factors in the biofilm formation process, the inhibition of this system may promote the eradication of biofilms by antimicrobial therapy [6]. The concentration of $15.6 \mu \mathrm{g} / \mathrm{ml}$ of quercetin significantly inhibited the biofilm formation in comparison with untreated PA01. Our findings are in accordance with those of Packiavathy et al [25] and Gopu et al [6] who reported that the biofilm formation in food-borne pathogens treated with $10 \mu \mathrm{g} / \mathrm{ml}$ of methyl eugenol or $40 \mu \mathrm{g} / \mathrm{ml}$ of quercetin was decreased in comparison with untreated control. According to our results, the combination of quercetin with imipenem or ciprofloxacin at the concentrations of 1/4 MIC showed the greatest inhibitory effect on biofilm formation with $84 \%$ reduction. In our previous studies, significant reduction of biofilm formation was also reported at sub-MIC concentrations of curcumin in combination with ciprofloxacin, ceftazidime, azithromycin and gentamicin [16, 17]. However, contrary to our findings, Shen et al [26] showed that the expression of some virulence factors in $P$. aeruginosa was increased at subinhibitory concentrations of vancomycin, tetracycline, ampicillin and azithromycin.

In our study, twitching and swarming motilities of $P$. aeruginosa PAO1 treated with 1/4 MIC of quercetin in combination with imipenem or ciprofloxacin was significantly impaired relative to untreated control. These results are consistent with reports which showed that curcumin [16, 17], salicylic acid [27], aspirin [7] and pyridoxal lactohydrazone [15] inhibit swarming and twitching motilities in $P$. aeruginosa.

Treated PAO1 cultures with 1/4 MIC of quercetin in combination with ciprofloxacin or imipenem showed the significant reduction in virulence factors including alginate and pyocyanin production and susceptibility to $\mathrm{H}_{2} \mathrm{O}_{2}$. Our results showed that these combinations significantly decreased the alginate (73.7-78.9\%) and pyocyanin (60.9-74.6\%) production compared with untreated PA01. Reduced alginate production was observed in food borne pathogens treated with $80 \mu \mathrm{g} / \mathrm{ml}$ of quercetin [6]. Also, Abraham et al [28] reported that Capparis spinosa decreased the alginate production up to $67 \%$ in Proteus mirabilis. Pyocyanin as an important virulence factor of $P$. aeruginosa inhibits the oxidative burst of phagocytic cells by apoptosis in host cells and effect to bacterial pathogenesis [15]. According to previous reports, pyocyanin inhibition showed the bactericidal activity and increased the susceptibility to oxygen free radicals. Similar to our results, the sub-MIC concentrations of aspirin [7], sodium ascorbate [29], pyridoxal lactohydrazone [15] and marine oligosaccharides [19] exhibited the inhibitory effects on pyocyanin production. In study conducted by Ouyang et al [4] quercetin demonstrated a remarkable ability to inhibit the production of pyocyanin, protease and elastase. However, they were not investigated the effect of 
quercetin on motility, alginate production and susceptibility to $\mathrm{H}_{2} \mathrm{O}_{2}$. We also confirmed that $1 / 4 \mathrm{MIC}$ of quercetin in combination with imipenem or ciprofloxacin significantly made bacterial strains more susceptible to $\mathrm{H}_{2} \mathrm{O}_{2}$. Similar results were reported by $\mathrm{He}$ et al [19] that demonstrated the feasibility of attenuating the tolerance of $P$. aeruginosa to azithromycin by using marine oligosaccharides. Increased $\mathrm{H}_{2} \mathrm{O}_{2}$ susceptibility was unexpected at first, as quercetin is known as important antioxidants $[11,12]$.

Our study indicated that quercetin alone and in combination significantly repressed the expression of las/ and lasR relative to untreated PA01. The combination of quercetin with imipenem or ciprofloxacin at the concentrations of 1/4 MIC showed the greatest reduction in the expression levels of las/and lasR. Considering the central role of quorum sensing in regulation of virulence factors and biofilm formation, we speculated that the quercetin mediated inhibition of these factors is achieved through effects on QS.

The docking results suggested that $\mathrm{H}$-bonding and hydrophobic interactions to amino acid residues between quercetin and binding sites play an important role in the conformer orientation and its stability in the active site of LasR and also can predict the molecular mechanism of QSI activity of quercetin in $P$. aeruginosa. According to in silico results, quercetin exhibited QSI activity and also showed high docking score when compared with 3-oxo- $\mathrm{C}_{12}$ - $\mathrm{HSL}$ native ligand in same docking conditions. The both of docked conformers of 3-oxo- $\mathrm{C}_{12}-\mathrm{HSL}$ and quercetin made $\mathrm{H}$-bond interactions with amino acid residues in active site of LasR which is similar to that reported for structure of regulator LasR bound to its autoinducer with amino acid residues of Tyr 56, Arg 61, Trp 60, Asp 73, Ser 129, and Thr 75 [30]. The H-bond interactions of phenolic moiety of quercetin had formed through hydroxyl groups with Leu 110 and Tyr 93 with distance of 2.03 and $2.13 \AA$, respectively. Also, oxygen atom of carbonyl group interacts with Arg 61 within distances of 2.52 and $2.14 \AA$. Furthermore, the other hydroxyl group next to carbonyl had formed hydrogen bond with Trp 60 with distance of $1.70 \AA$ in the active site of LasR. In previous studies, anti-QS activity of various synthetic and natural compounds against $P$. aeruginosa was proved through docking analysis $[6,7,15,31]$.

To our knowledge, this is the first study to show additive activity of sub-MIC concentrations of quercetin with imipenem or ciprofloxacin against $P$. aeruginosa QS related genes and virulence factors. Gopu et al [6] investigated the synergistic activity of quercetin with erythromycin, tetracycline, kanamycin, gentamycin, ampicillin, and chloramphenicol in food borne pathogens. In checkerboard titration, quercetin reduced the MICs of erythromycin, tetracycline, and chloramphenicol in $P$. aeruginosa strain PUFSTb04. However, there was not synergistic activity between $40 \mu \mathrm{g} / \mathrm{ml}$ of quercetin with these antibiotics in PUFSTb0 strain.

\section{Conclusions}

Our results indicate the potential of a combination of quercetin with imipenem or ciprofloxacin at the concentrations of $1 / 4 \mathrm{MIC}$ to inhibit biofilm formation and QS related genes and virulence traits. Considering the beneficial biological effects of quercetin and its inhibitory effects on biofilm formation and QS related virulence traits at low concentrations $(15.6$ and $3.9 \mu \mathrm{g} / \mathrm{ml})$, the combination of this natural 
compound with imipenem or ciprofloxacin may be used as an effective approach for managing of multidrug resistant infections of $P$. aeruginosa.

\section{Abbreviations}

Q: Quercetin; IPM: Imipenem; CIP: Ciprofloxacin; QS: quorum sensing; MDR: Multidrug resistant; AHL: Nacyl homoserine lactones; QSIs: Quorum sensing inhibitors; Sub-MIC: Subinhibitory concentrations; FIC: Fractional inhibitory concentration.

\section{Declarations}

\section{Acknowledgements}

The authors would like to acknowledge Zanjan University of Medical Sciences for supporting our study (A-12-535-23, IR.ZUMS.REC.1397.339).

\section{Funding}

Not applicable

\section{Availability of data and material}

All data generated or analysed during this study are included in this published article.

\section{Authors' contributions}

TG, FR and MG involved in the project development, data management. AH participated in project development, data analysis. $\mathrm{FH}$ and $\mathrm{HZ}$ involved in the project development, data management and manuscript writing. All authors have read and approved the final version of manuscript.

\section{Ethics approval and consent to participate}

The ethical clearance (IR.ZUMS.REC.1397.339) was obtained from the research ethics committee of Zanjan University of Medical Sciences, Zanjan, Iran.

\section{Consent for publication}

Not applicable.

\section{Competing interests}

The authors declare that they have no competing interests.

\section{References}


1. Matos ECO, Andriolo RB, Rodrigues YC, Lima PDL, Carneiro I, Lima KVB: Mortality in patients with multidrug-resistant Pseudomonas aeruginosa infections: a meta-analysis. Revista da Sociedade Brasileira de Medicina Tropical 2018, 51(4):415-420.

2. Raman G, E. Avendano E, Chan J, Merchant S, Puzniak L: Risk factors for hospitalized patients with resistant or multidrug-resistant Pseudomonas aeruginosa infections: A systematic review and metaanalysis, vol. 7; 2018.

3. Manner S, Fallarero A: Screening of Natural Product Derivatives Identifies Two Structurally Related Flavonoids as Potent Quorum Sensing Inhibitors against Gram-Negative Bacteria. International journal of molecular sciences 2018, 19(5).

4. Ouyang J, Sun F, Feng W, Sun Y, Qiu X, Xiong L, Liu Y, Chen Y: Quercetin is an effective inhibitor of quorum sensing, biofilm formation and virulence factors in Pseudomonas aeruginosa. Journal of applied microbiology 2016, 120(4):966-974.

5. Olivero-Verbel J, Barreto-Maya A, Bertel-Sevilla A, Stashenko EE: Composition, anti-quorum sensing and antimicrobial activity of essential oils from Lippia alba. Braz J Microbiol 2014, 45(3):759-767.

6. Gopu V, Meena CK, Shetty PH: Quercetin Influences Quorum Sensing in Food Borne Bacteria: In-Vitro and In-Silico Evidence. PloS one 2015, 10(8):e0134684.

7. El-Mowafy SA, Abd El Galil KH, El-Messery SM, Shaaban MI: Aspirin is an efficient inhibitor of quorum sensing, virulence and toxins in Pseudomonas aeruginosa. Microbial pathogenesis 2014, 74:25-32.

8. Chan K-G, Liu Y-C, Chang C-Y: Inhibiting N-acyl-homoserine lactone synthesis and quenching Pseudomonas quinolone quorum sensing to attenuate virulence. Front Microbiol 2015, 6:1173-1173.

9. Vasavi HS, Arun AB, Rekha PD: Anti-quorum sensing activity of flavonoid-rich fraction from Centella asiatica L. against Pseudomonas aeruginosa PA01. Journal of microbiology, immunology, and infection = Wei mian yu gan ran za zhi 2016, 49(1):8-15.

10. Starkey M, Lepine F, Maura D, Bandyopadhaya A, Lesic B, He J, Kitao T, Righi V, Milot S, Tzika A et al: Identification of anti-virulence compounds that disrupt quorum-sensing regulated acute and persistent pathogenicity. PLoS pathogens 2014, 10(8):e1004321.

11. Erdönmez D, Yousefı Rad A, Aksöz N: Anti-Quorum Sensing Potential of Antioxidant Quercetin and Resveratrol, vol. 61; 2018.

12. Paczkowski JE, Mukherjee S, McCready AR, Cong JP, Aquino CJ, Kim H, Henke BR, Smith CD, Bassler BL: Flavonoids Suppress Pseudomonas aeruginosa Virulence through Allosteric Inhibition of Quorum-sensing Receptors. The Journal of biological chemistry 2017, 292(10):4064-4076.

13. Laboratory Standards Institute antimicrobial susceptibility testing standards, vol. M02-A12, M07-A10, and M11-A8. 2017; 2017.

14. Bonapace CR, Bosso JA, Friedrich LV, White RL: Comparison of methods of interpretation of checkerboard synergy testing. Diagnostic microbiology and infectious disease 2002, 44(4):363-366.

15. Heidari A, Noshiranzadeh N, Haghi F, Bikas R: Inhibition of quorum sensing related virulence factors of Pseudomonas aeruginosa by pyridoxal lactohydrazone. Microbial pathogenesis 2017, 112:103- 
110.

16. Roudashti S, Zeighami H, Mirshahabi H, Bahari S, Soltani A, Haghi F: Synergistic activity of subinhibitory concentrations of curcumin with ceftazidime and ciprofloxacin against Pseudomonas aeruginosa quorum sensing related genes and virulence traits, vol. 33; 2017.

17. Bahari S, Zeighami H, Mirshahabi H, Roudashti S, Haghi F: Inhibition of Pseudomonas aeruginosa quorum sensing by subinhibitory concentrations of curcumin with gentamicin and azithromycin. Journal of global antimicrobial resistance 2017, 10:21-28.

18. Hoffmann N, Lee B, Hentzer M, Rasmussen TB, Song Z, Johansen HK, Givskov M, Hoiby N: Azithromycin blocks quorum sensing and alginate polymer formation and increases the sensitivity to serum and stationary-growth-phase killing of Pseudomonas aeruginosa and attenuates chronic $P$. aeruginosa lung infection in Cftr(-/-) mice. Antimicrobial agents and chemotherapy 2007, 51(10):3677-3687.

19. He X, Hwang HM, Aker WG, Wang P, Lin Y, Jiang X, He X: Synergistic combination of marine oligosaccharides and azithromycin against Pseudomonas aeruginosa. Microbiological research 2014, 169(9-10):759-767.

20. Livak KJ, Schmittgen TD: Analysis of relative gene expression data using real-time quantitative PCR and the 2(-Delta Delta C(T)) Method. Methods (San Diego, Calif) 2001, 25(4):402-408.

21. Morris GM, Huey R, Lindstrom W, Sanner MF, Belew RK, Goodsell DS, Olson AJ: AutoDock4 and AutoDockTools4: Automated docking with selective receptor flexibility. Journal of computational chemistry 2009, 30(16):2785-2791.

22. Kramer B, Rarey M, Lengauer T: Evaluation of the FLEXX incremental construction algorithm for protein-ligand docking. Proteins: Structure, Function, and Bioinformatics 1999, 37(2):228-241.

23. Wang X, Cai Y, Xing H, Wu W, Wang G, Li L, Chen J: Increased therapeutic efficacy of combination of azithromycin and ceftazidime on Pseudomonas aeruginosa biofilm in an animal model of ureteral stent infection. BMC microbiology 2016, 16(1):124.

24. Tang K, Zhang X-H: Quorum quenching agents: resources for antivirulence therapy. Mar Drugs 2014, 12(6):3245-3282.

25. Abraham Sybiya Vasantha Packiavathy I, Agilandeswari P, Syed Musthafa K, Pandian S, Arumugam VR: Antibiofilm and quorum sensing inhibitory potential of Cuminum cyminum and its secondary metabolite methyl eugenol against Gram negative bacterial pathogens, vol. 45; 2012.

26. Shen L, Shi Y, Zhang D, Wei J, Surette MG, Duan K: Modulation of secreted virulence factor genes by subinhibitory concentrations of antibiotics in Pseudomonas aeruginosa. Journal of microbiology (Seoul, Korea) 2008, 46(4):441-447.

27. Guo M, Gamby S, Zheng Y, Sintim HO: Small molecule inhibitors of Al-2 signaling in bacteria: stateof-the-art and future perspectives for anti-quorum sensing agents. International journal of molecular sciences 2013, 14(9):17694-17728.

28. Issac Abraham SV, Palani A, Ramaswamy BR, Shunmugiah KP, Arumugam VR: Antiquorum sensing and antibiofilm potential of Capparis spinosa. Archives of medical research 2011, 42(8):658-668. 
29. El-Mowafy SA, Shaaban Ml, Abd El Galil KH: Sodium ascorbate as a quorum sensing inhibitor of Pseudomonas aeruginosa. Journal of applied microbiology 2014, 117(5):1388-1399.

30. Bottomley MJ, Muraglia E, Bazzo R, Carfi A: Molecular insights into quorum sensing in the human pathogen Pseudomonas aeruginosa from the structure of the virulence regulator LasR bound to its autoinducer. The Journal of biological chemistry 2007, 282(18):13592-13600.

31. Heidari A, Haghi F, Noshiranzadeh N, Bikas R: (S,E)-2-hydroxy-N-(2-hydroxy-5-nitrobenzylidene) propane hydrazide as a quorum sensing inhibitor of Pseudomonas aeruginosa; 2017.

\section{Figures}

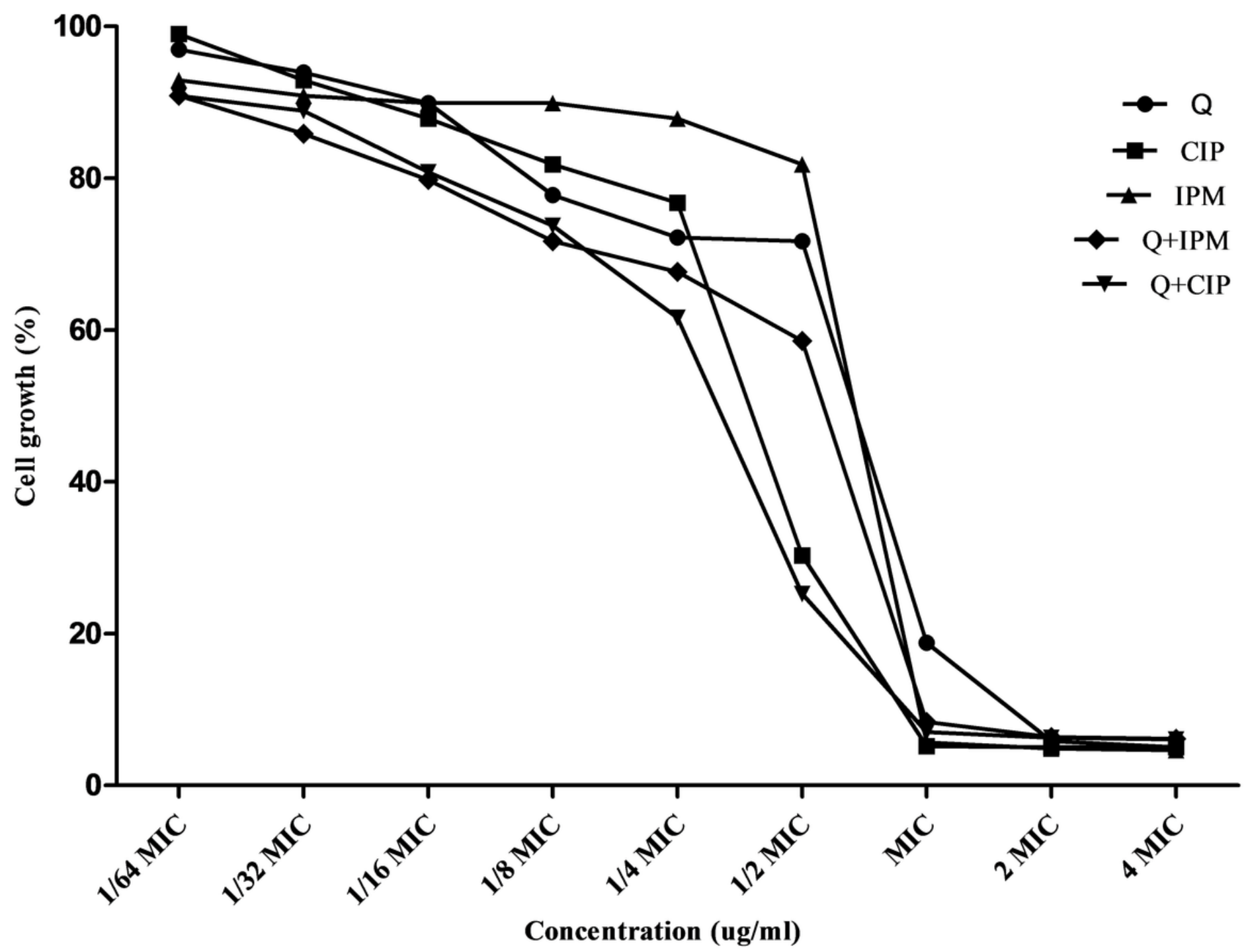

Figure 1

The effect of concentrations of $0.015 \times$ MIC to $4 \times$ MIC of quercetin, imipenem and ciprofloxacin alone and in combination on cell growth of PAO1. 


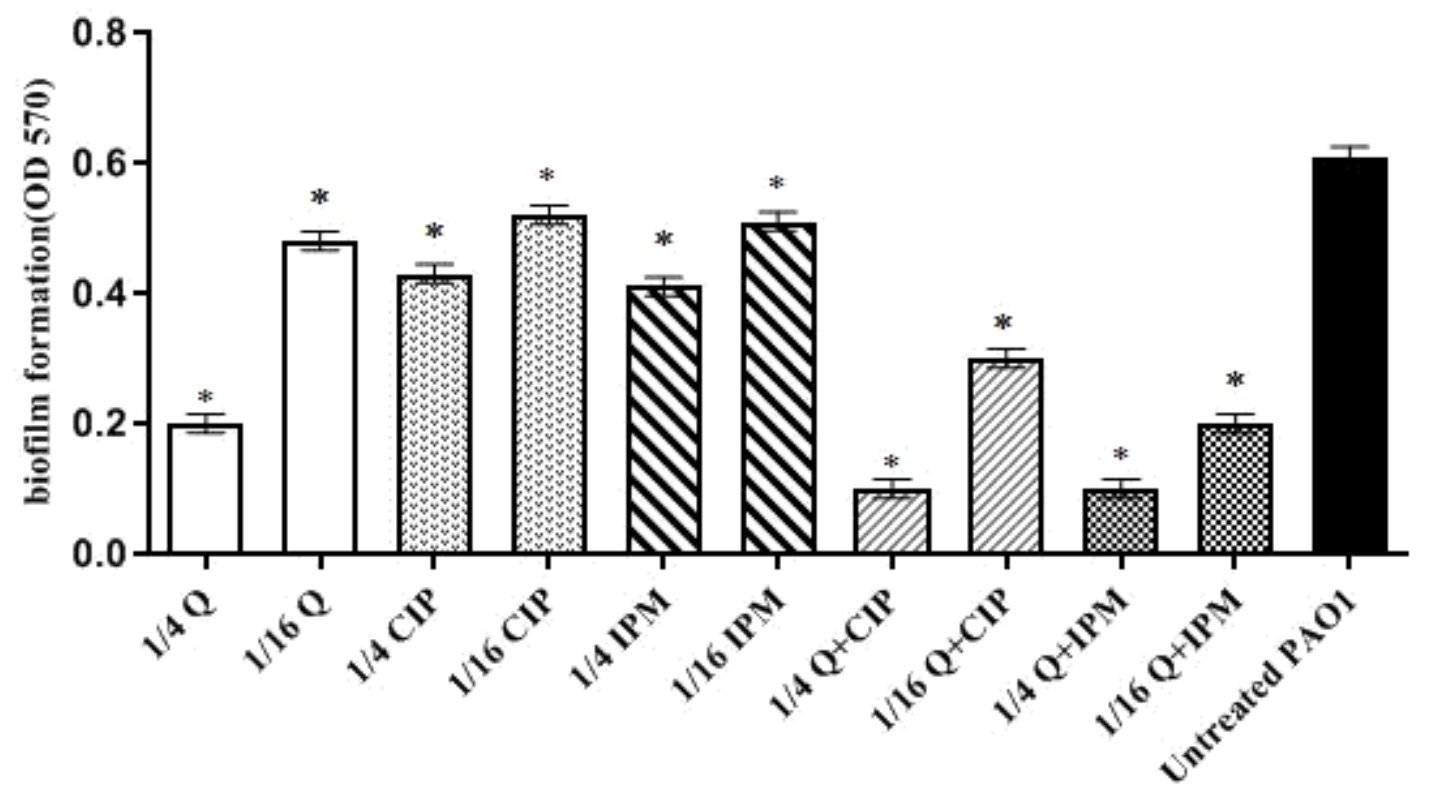

Figure 2

Effect of subinhibitory concentrations (1/4 and 1/16 MIC) of quercetin (Q), ciprofloxacin (CIP) and imipenem (IPM) alone and in combination on biofilm formation ( ${ }^{*}$, significant, $\left.\mathrm{P}<0.05\right)$.

$\infty$ Swarming

$\square$ Twitching

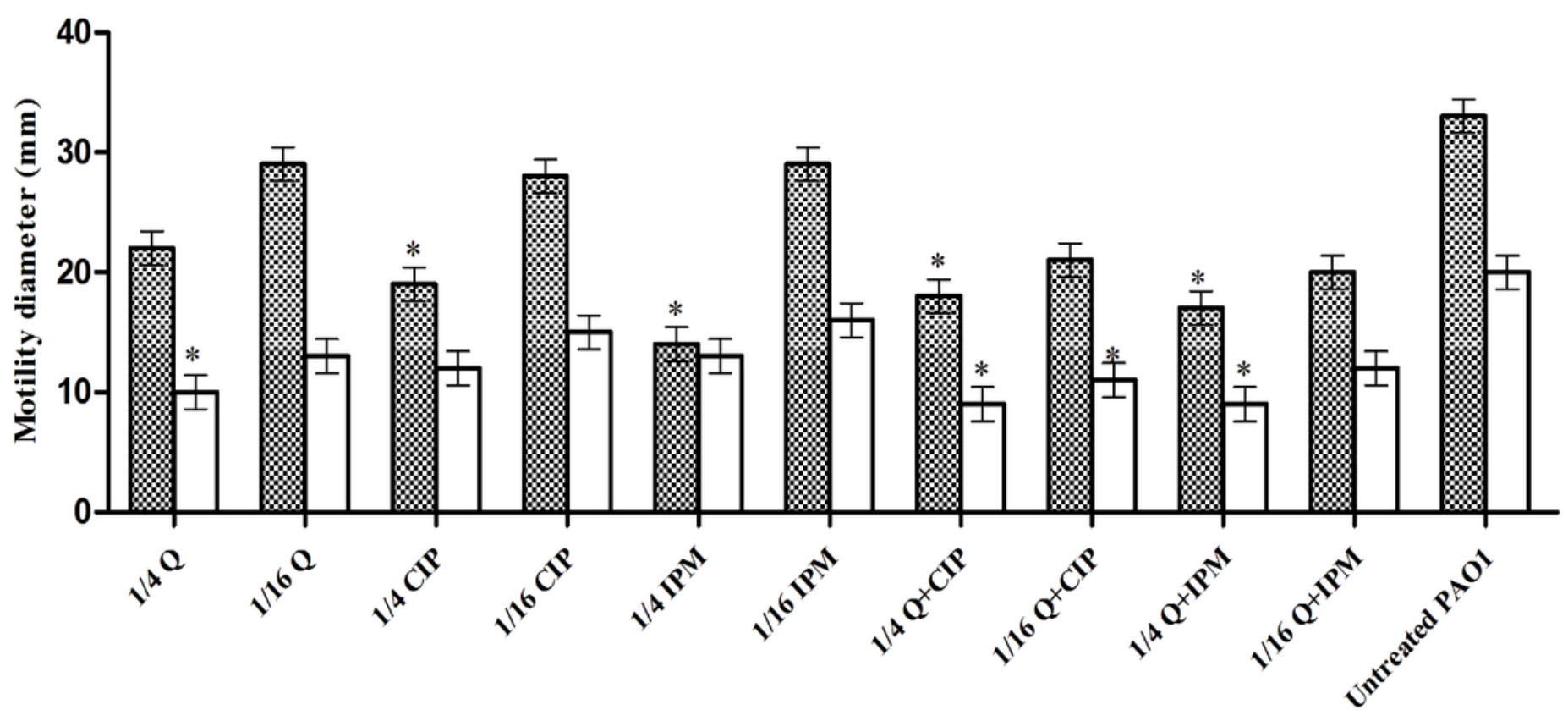

Figure 3 
Effect of subinhibitory concentrations (1/4 and 1/16 MIC) of quercetin (Q), ciprofloxacin (CIP) and imipenem (IPM) alone and in combination on the swarming $\otimes$ and twitching $\otimes$ motilities $\left({ }^{*}\right.$, significant, $\mathrm{P}<$ 0.05).

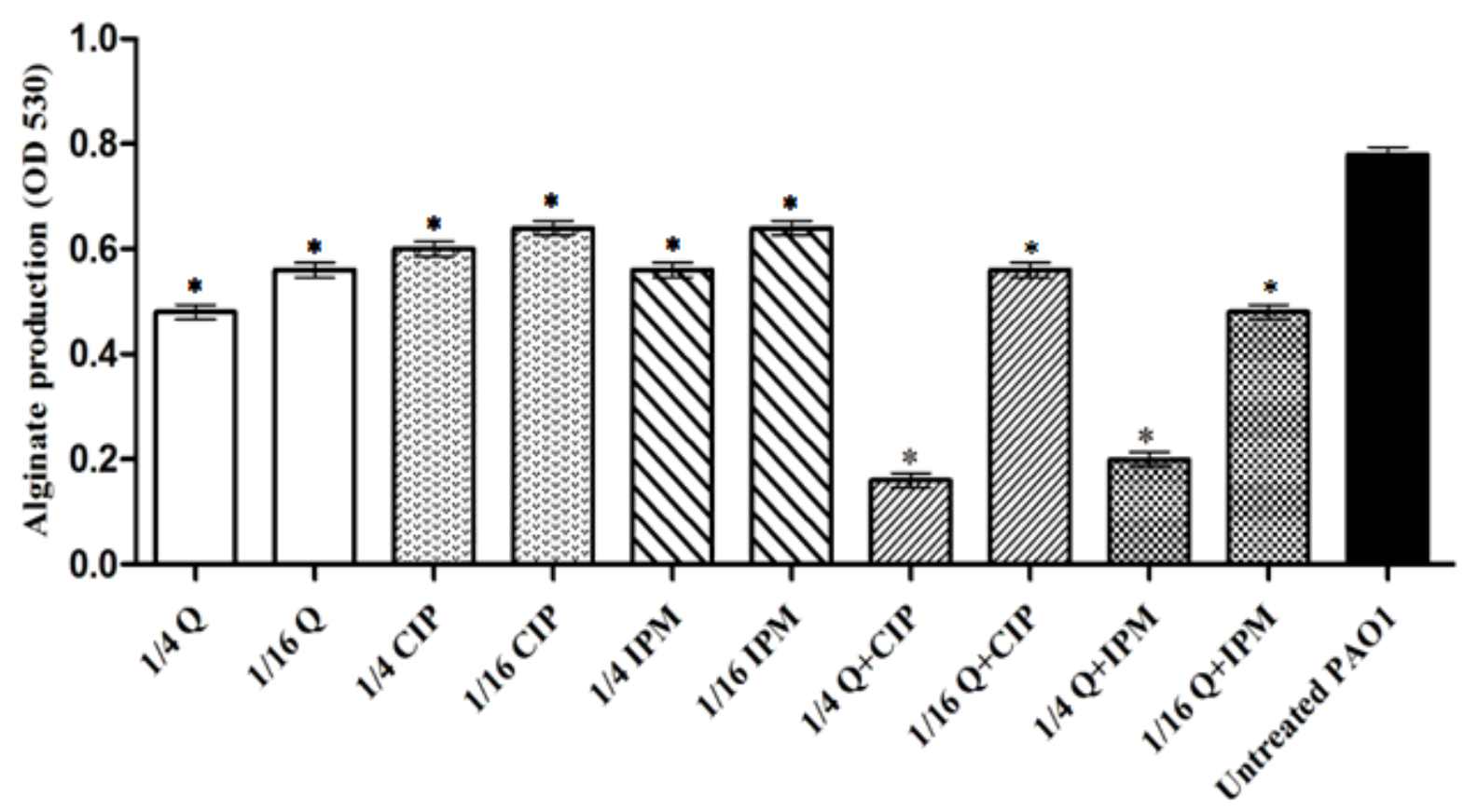

Figure 4

Effect of subinhibitory concentrations (1/4 and 1/16 MIC) of quercetin (Q), ciprofloxacin (CIP) and imipenem (IPM) alone and in combination on the alginate production $\left({ }^{*}\right.$, significant, $\left.\mathrm{P}<0.05\right)$. 


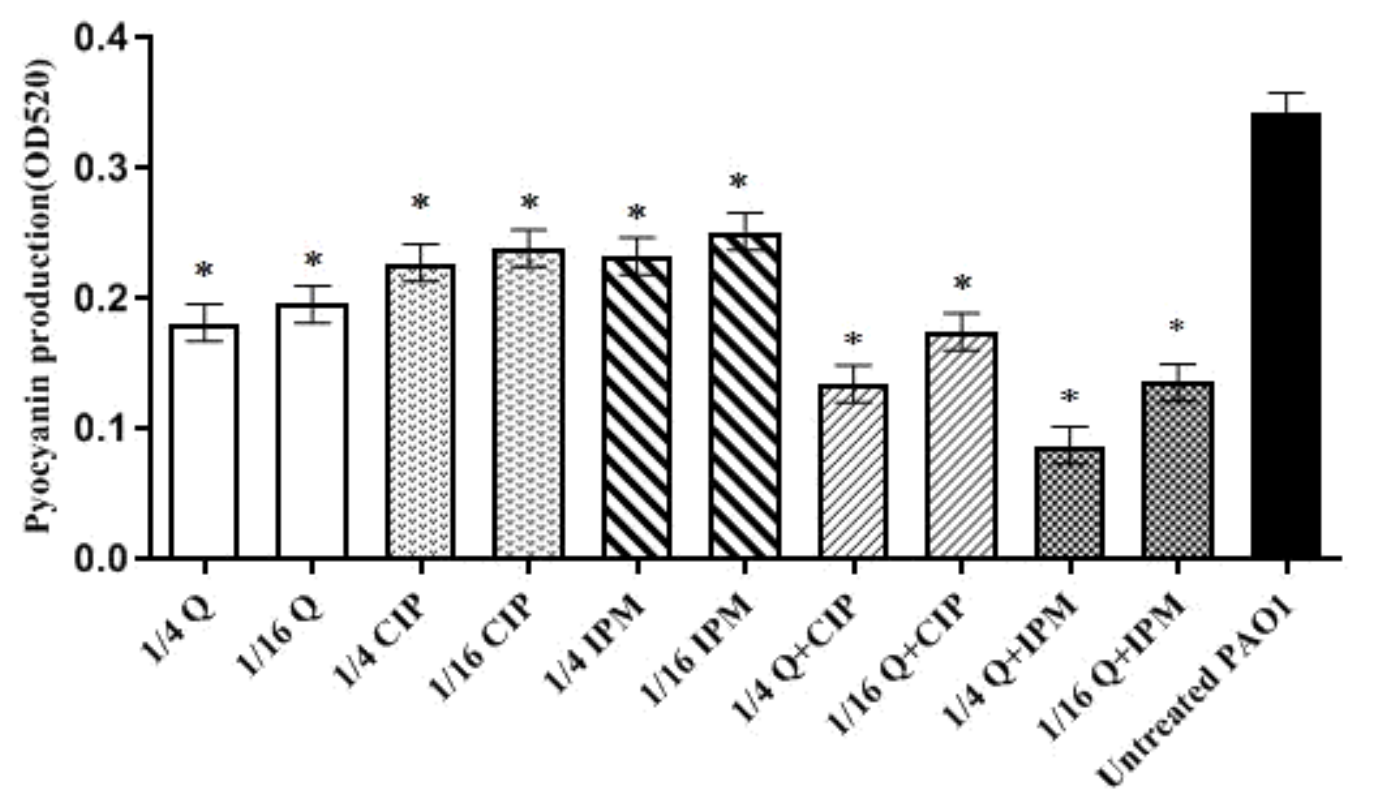

Figure 5

Effect of subinhibitory concentrations (1/4 and 1/16 MIC) of quercetin (Q), ciprofloxacin (CIP) and imipenem (IPM) alone and in combination on pyocyanin production $\left({ }^{*}\right.$, significant, $\left.P<0.05\right)$.

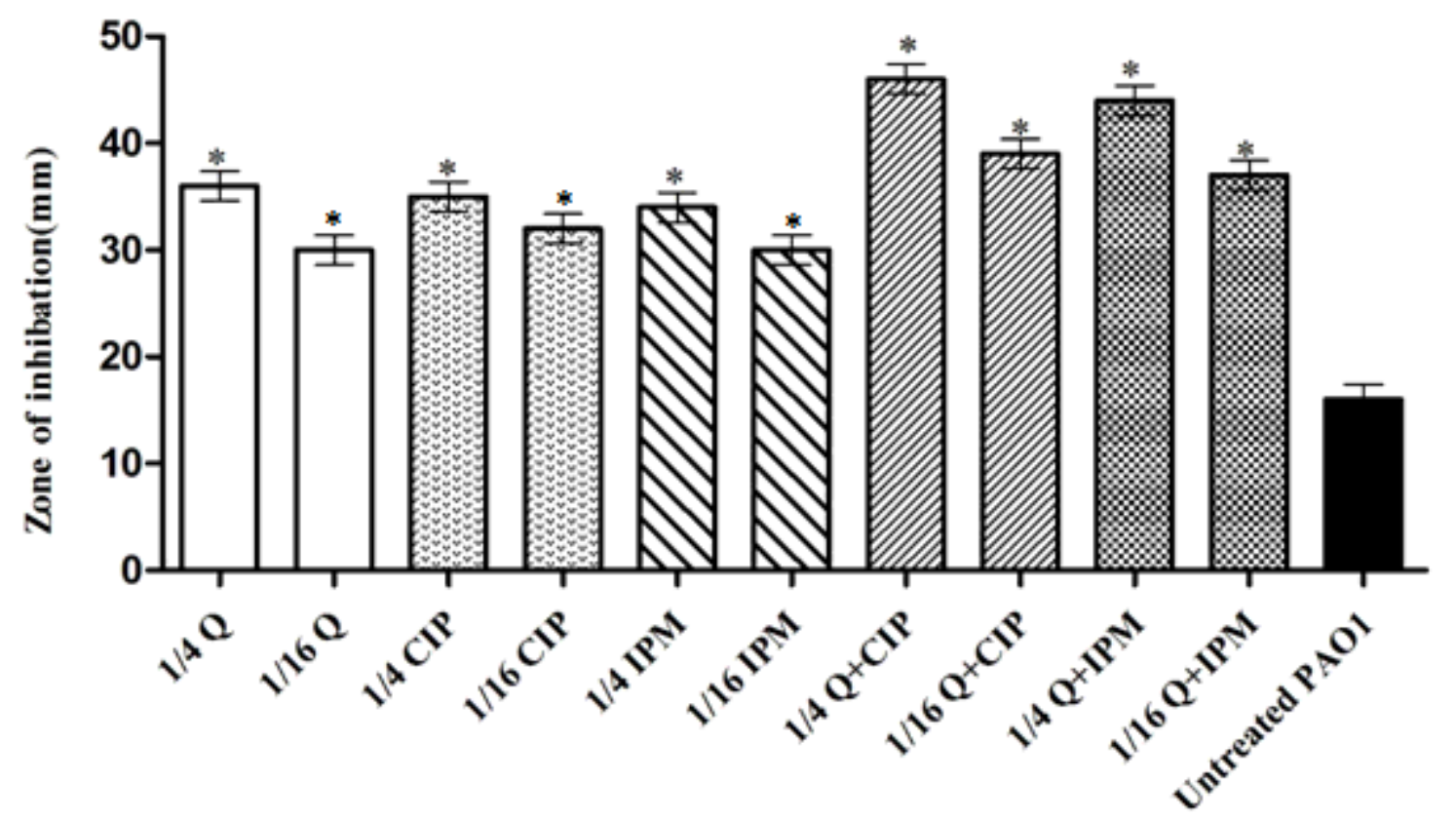

Figure 6 
Effect of subinhibitory concentrations (1/4 and 1/16 MIC) of quercetin (Q), ciprofloxacin (CIP) and imipenem (IPM) alone and in combination on $\mathrm{H} 2 \mathrm{O} 2$ susceptibility $\left({ }^{*}\right.$, significant, $\left.\mathrm{P}<0.05\right)$.

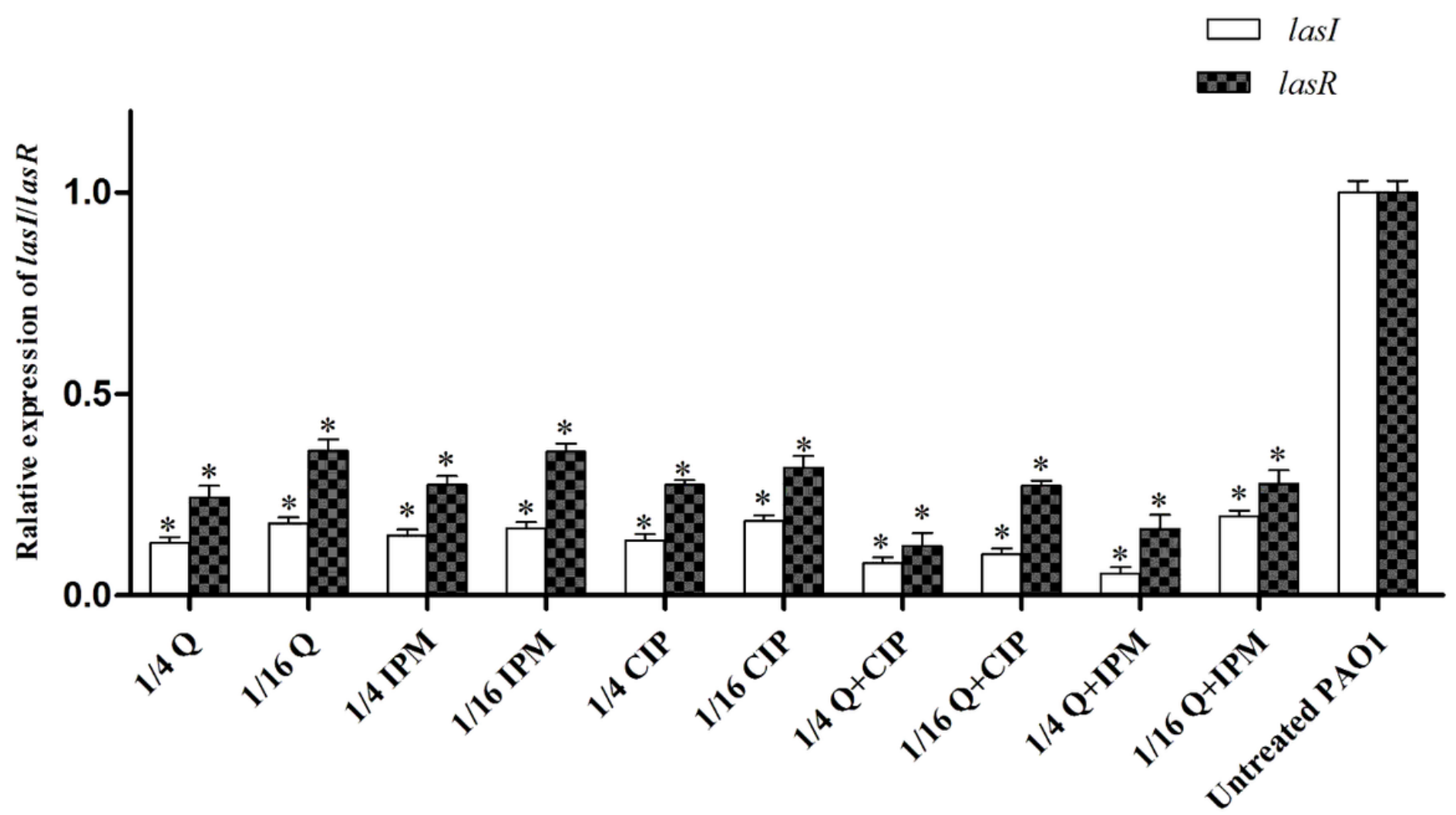

Figure 7

Quercetin (Q), ciprofloxacin (CIP) and imipenem (IPM) at subinhibitory concentrations (1/4 and 1/16 MIC) alone and in combination inhibited QS regulated genes in treated PAO1 ( ${ }^{*}$, significant, $\left.\mathrm{P}<0.05\right)$. 


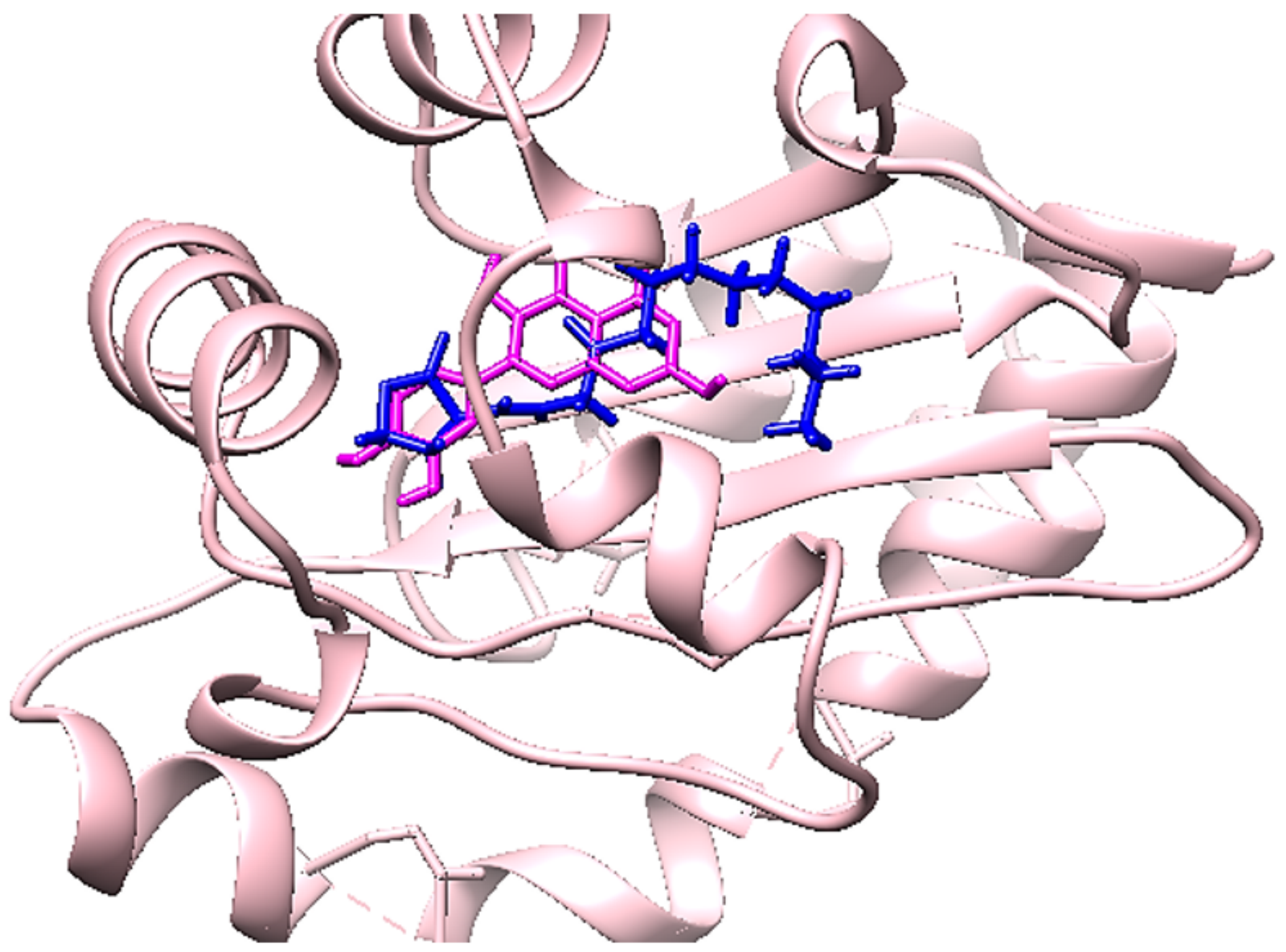

Figure 8

(a) Ribbon view of docked conformer of Quercetin (pink colour stick model) and (b) hydrogen-bonding interactions between the docked conformer of Quercetin and amino acids in the active site of LasR. 


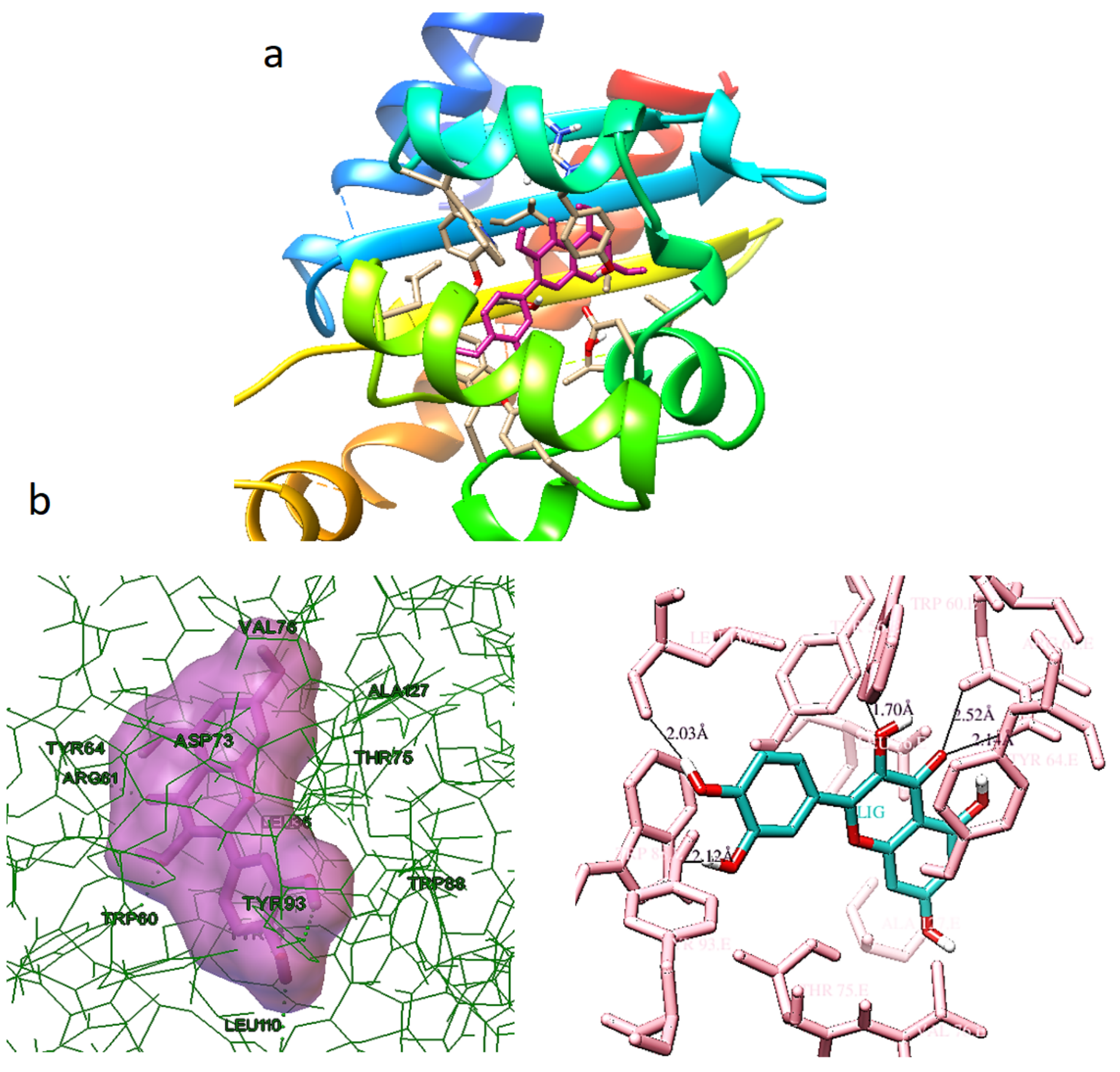

Figure 9

Overlay of the Quercetin (pink colour stick model) with crystal ligand 3-oxo-C12-HSL (blue colour stick model) in the active site of LasR. 